\title{
PN-TRACE: PLAIN NETWORK-WIDE BROADCASTING THROUGH TIME RESERVATION USING ADAPTIVE CONTROL FOR ENERGY EFFICIENCY
}

\author{
Bulent Tavli and Wendi B. Heinzelman \\ Department of Electrical and Computer Engineering, \\ University of Rochester, Rochester NY
}

Abstract- In this paper, we present Plain Network-wide broadcasting through Time Reservation using Adaptive Control for Energy efficiency (PN-TRACE), which is an energy-efficient network-wide voice broadcasting architecture for mobile ad hoc networks. PN-TRACE is created by integrating flooding, which is a simple, stateless broadcast routing protocol, and Multi-Hop Time Reservation using Adaptive Control for Energy Efficiency (MH-TRACE), which is an energy conserving MAC protocol designed to provide QoS for voice traffic. To evaluate the performance of PN-TRACE in terms of energy dissipation and QoS, we conducted simulations with a 41 node, 6-hop network using a $32 \mathrm{kbps}$ voice source. We compared PN-TRACE with flooding using the IEEE 802.11 and SMAC medium access control protocols. Simulation results show that all schemes give similar QoS performances under the same settings. However, the energy dissipation of PN-TRACE is less than $18 \%$ of the energy dissipation of IEEE 802.11-based flooding due to $P N-T R A C E$ 's energy conserving design. The energy saving of SMAC-based flooding is not significant due to the limited sleep period.

\section{INTRODUCTION}

\section{Motivation}

One of the most important functions of a mobile ad-hoc radio network in military applications is to create a platform for voice communications. Due to the limited radio range, single hop broadcasting is not possible in many battlefield scenarios, and thus multi-hop broadcasting is unavoidable. Although multi-hop broadcasting is a commonly employed network service, it can also be used as a standalone service, especially for voice communications with stringent quality of service (QoS) requirements.

In network-wide voice broadcasting we have two main criteria to evaluate the performance of the network architecture: application QoS and energy efficiency. QoS for voice communications requires that (i) the maximum packet delay is kept within specific bounds and (ii) the packet delivery ratio is high. Energy efficiency is crucial because soldiers are equipped with short-range lightweight radios operating with limited energy. Avoiding the energy waste for these radios is of the utmost importance in order to keep the soldiers connected to the network.

\section{QoS Bounds for Voice Traffic}

QoS for streaming media necessitates timely delivery of packets (bounded delay) and high packet delivery ratio. In broadcasting scenarios, where acknowledged data delivery is not practical, QoS of the streaming media is determined primarily by the MAC layer. One solution to meet the delay and packet delivery requirements for voice is to use periodic time-frame based medium access with automatic renewal of channel access, where the frame rate is matched to the periodic rate of the voice sources [1]. This ensures that flows are uninterrupted, but it requires central control to coordinate channel access. Although it is quite straightforward to coordinate channel access in single-hop networks, regulating and optimizing channel access with partial information about the network status is a challenging task in multi-hop networks.

\section{Energy Dissipation}

Avoiding energy waste is crucial in order to keep the nodes connected to the network. Energy efficiency can be achieved by (i) optimizing the transmit power, which is proportional to the transmit range, (ii) minimizing the idle energy dissipation, which means maximizing the sleep time, (iii) avoiding overhearing irrelevant packets, (iv) avoiding unnecessary carrier sensing and (v) reducing the overhead as much as possible without sacrificing the robustness and fault tolerance of the network.

It has been shown that optimal network-wide broadcast scheduling for throughput optimization in a multi-hop, mobile, packet radio network is NP-complete [2]. Furthermore, it remains as an open question whether minimum transmit energy broadcast routing can be solved in polynomial time, despite the NP-hardness of its general graph version [3].

By considering both transmit and receive energy dissipation, it has been shown that for a given energy and propagation model there is an optimum transmit radius, $\operatorname{Tr}_{O P}$, beyond which single hop transmission is less energy efficient than multi-hop transmissions [4]. Thus, the optimal broadcast strategy to minimize energy dissipation is to use a multi-hop broadcasting scheme, where the transmit radius is chosen as $\operatorname{Tr}_{O P}$.

Avoiding energy dissipation in the idle mode necessitates coordination through scheduling between the nodes, so that nodes avoid idle listening or overhearing 
irrelevant packets or collisions, especially in broadcasting scenarios. While this goal can be accomplished using centralized control, this is not practical in a mobile ad hoc network, or at least not scalable due to the high overhead to monitor and convey the control information throughout the network. Network partitioning through clustering introduces a realizable, yet useful framework for network coordination, which has been investigated thoroughly [5].

The remainder of this paper is organized as follows. Section II describes the broadcast architectures evaluated in this paper. These broadcast architectures are IEEE 802.11-based flooding, Sensor MAC (SMAC)-based flooding, and Plain Network-wide Broadcasting through Time Reservation using Adaptive Control for Energy efficiency (PN-TRACE). The simulation environment and results are presented in Section III. Conclusions are drawn in Section IV, and Section V addresses our future work.

\section{BROADCAST ARCHITECTURES}

We evaluated the performance of three network-wide broadcast architectures. These architectures are IEEE 802.11-based flooding, SMAC-based flooding, and PN-TRACE. We obtained quantitative comparisons of PNTRACE, 802.11-based flooding and SMAC-based flooding for various metrics. There are two main reasons to compare PN-TRACE with IEEE 802.11 and SMACbased flooding: (i) Both of these protocols are well known by the wireless community, and almost all researchers compare their algorithms with IEEE 802.11, making it possible to compare PN-TRACE with any other protocol by just comparing the performance relative to IEEE 802.11, and (ii) SMAC is the most prominent example of a truly distributed energy aware MAC protocol. In this section we provide brief descriptions of these architectures.

\section{Flooding}

Flooding is the simplest broadcasting algorithm, where each node rebroadcasts every packet it receives for the first time [6]. Each node keeps track of the packets it received (i.e., the source node ID and packet sequence number given by the source creates a unique global ID for each packet), and duplicate rebroadcasts are avoided. Flooding is also a stateless algorithm, so the nodes do not need to create a routing framework (e.g., routing tables, gateways, route caching, etc.). Due to the advantageous features of flooding, many unicasting protocols use flooding as a means for network-wide broadcasting for route discovery [8]. However, flooding has some drawbacks, such as broadcast storms (excessive redundant rebroadcasts) that use up the available bandwidth quickly [7]. Furthermore, flooding inherently is very inefficient due to its redundant structure. Especially for large networks (e.g., larger than a few hops) and high node densities (e.g., more than 10 neighbors per node), flooding is an unstable algorithm.

\section{IEEE 802.11-based Broadcasting}

In broadcasting mode, IEEE 802.11 uses $p$-persistent CSMA with a constant defer window length (i.e., the default minimum defer period) [10]. When a node has a packet to broadcast, it picks a random defer time and starts to sense the channel. When the channel is sensed idle the defer timer counts down from the initially selected defer time at the end of each time slot. When the channel is sensed busy, the defer timer is not decremented. Upon the expiration of the defer timer the packet is broadcast.

However, when performing network-wide flooding, the contention resolution algorithm of IEEE 802.11 cannot successfully avoid collisions due to the high number of nodes contending for channel access concurrently. One method to avoid this problem is to spread out the packet transmissions at a higher level (e.g., the network layer) by applying a random delay chosen from a uniform distribution between $\left[0, T_{\text {spread }}\right]$. In our simulations we used $T_{\text {spread }}$ as $12.5 \mathrm{~ms}$, which gives the best packet delivery ratio.

\section{SMAC-based Broadcasting}

SMAC is an energy-efficient MAC protocol built on top of IEEE 802.11 [11]. The basic design philosophy of SMAC is to turn the radios off periodically to save energy that would be wasted in idle mode. The original protocol, designed for low bandwidth unicast traffic in sensor networks, is shown to be highly energy efficient [11].

We modified the original SMAC protocol to compare it with the other MAC protocols on a fair basis. Actually, we take the basic design philosophy of SMAC, which is letting the nodes sleep periodically to save energy, and modified 802.11 to create the modified SMAC. Since we assumed global synchronization for all the MAC protocols, we also assumed global synchronization for SMAC, so there are no synchronization packets and overhead in the modified SMAC. We tested several sleep/awake ratios, and the optimal schedule (i.e., highest throughput) for SMAC is a 25 ms sleep/awake cycle with $15 \%$ sleep time. Since the node density and packet generation rate in our framework is much higher than the cases tested in [11], several modifications are needed to optimize SMAC, like randomization of the contention start time after the sleep period for the packets that could not be transmitted in the previous awake period. If all the nodes with stored packets begin contention at the beginning of the awake period, almost all the packets would collide, because it is not possible to comply with such high medium access demand 
at once for the underlying 802.11 contention resolution algorithm.

\section{PN-TRACE}

Plain Network-wide broadcasting through multi-hop Time Reservation using Adaptive Control for Energy Efficiency (PN-TRACE) is created by an integrated cross layer combination of Multi-Hop Time Reservation Using Adaptive Control for Energy Efficiency (MH-TRACE) [9] as the MAC layer and flooding as the routing layer. Figure 1 shows a snapshot of MH-TRACE clustering and medium access for a portion of a distribution of mobile nodes. In MH-TRACE, the network is partitioned into overlapping clusters through a distributed algorithm. Time is organized into cyclic constant duration superframes consisting of several frames. Each clusterhead chooses the least noisy frame to operate within and dynamically changes its frame according to the interference level of the dynamic network. Nodes gain channel access through a dynamically updated and monitored transmission schedule created by the clusterheads, which eliminates packet collisions within the cluster. Collisions with the members of other clusters are also minimized by the clusterhead's selection of the minimal interference frame.

Ordinary nodes are not static members of clusters, but they choose the cluster they want to join based on the spatial and temporal characteristics of the traffic, taking into account the proximity of the clusterheads and the availability of the data slots within the corresponding cluster. Each frame consists of a control sub-frame for

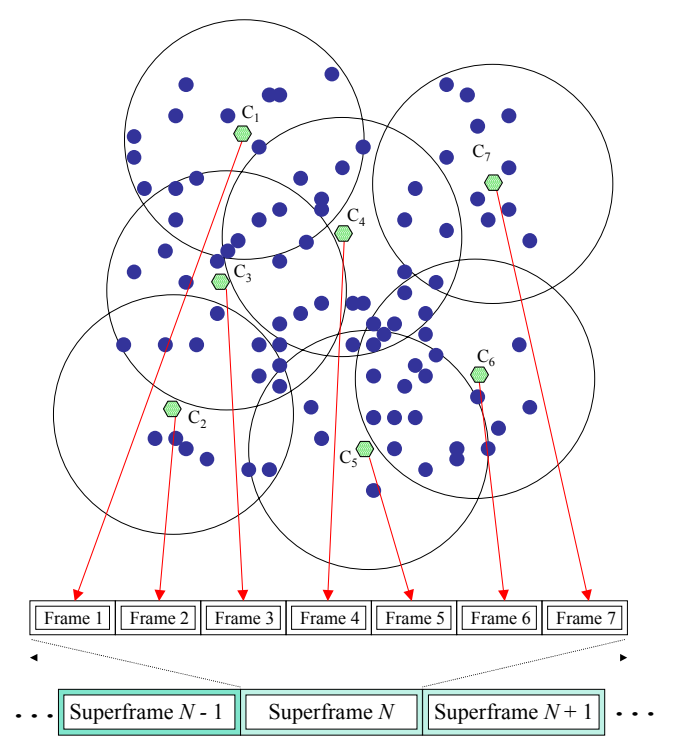

Figure 1. A snapshot of MH-TRACE clustering and medium access for a portion of an actual distribution of mobile nodes. Nodes $\mathrm{C}_{1}-\mathrm{C}_{7}$ are clusterhead nodes.

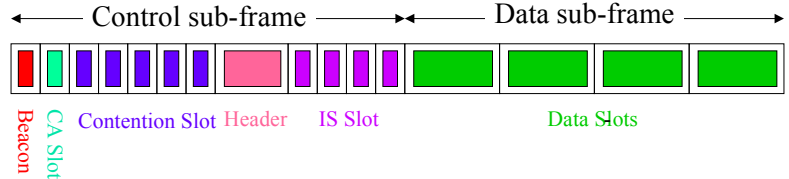

Figure 2. MH-TRACE frame structure.

transmission of control packets, and a contention-free data sub-frame for data transmission (see Figure 2). Beacon packets are used for the announcement of the start of a new frame; Clusterhead Announcement (CA) packets are used for reducing co-frame cluster interference; contention slots are used for initial channel access requests; the header packet is used for announcing the data transmission schedule for the current frame; and Information Summarization (IS) packets are used for announcing the upcoming data packets. IS packets are designed to be versatile, and they are crucial in energy saving. Each scheduled node transmits its data at the reserved data slot.

Nodes that are scheduled to transmit data send a short information summarization (IS) packet prior to data transmission. The IS packet includes information about the data packet, where the content of the IS packets can be modified to fit the requirements of different applications. In PN-TRACE, we include the source ID and the packet sequence number in the IS packet, so that nodes that already received a particular data packet avoid receiving a duplicate of the same packet, which saves a considerable amount of energy.

There are several mechanisms in PN-TRACE that provide energy efficiency: (i) nodes are in the sleep mode whenever they are not involved in data transmission or reception, which saves the energy that would be wasted in idle mode or in carrier sensing, and (ii) nodes can selectively choose what data to receive based on information from the IS packets, enabling the nodes to avoid receiving redundant data (i.e., multiple receptions of the same packet).

\section{SIMULATIONS}

To test the performance of the three broadcast architectures, we ran simulations using the ns-2 simulator. The channel rate is set to 2 Mbps. We simulated conversational voice coded at $32 \mathrm{Kbps}$ with 100-byte payload data packets, which corresponds to one voice packet per $25 \mathrm{~ms}$. Data packet overhead is 10 bytes for IEEE 802.11-based flooding, SMAC-based flooding, and PN-TRACE. PN-TRACE control packets are 10 bytes, except the header packet, which is 22 bytes. All time slots are separated by one Inter-Frame Space (IFS) time, which is $16 \mu \mathrm{s}$. In PN-TRACE, there are 6 frames within the 
Table I. Simulation parameters.

\begin{tabular}{lll}
\hline Var. & Description & Value \\
\hline$N_{N}$ & Number of nodes & 41 \\
$D_{T r}$ & Trans. range & $250 \mathrm{~m}$ \\
$D_{C S}$ & CS range & $507 \mathrm{~m}$ \\
$T_{\text {drop }}$ & Pck. drop thresh. & $150 \mathrm{~ms}$ \\
$P_{T}$ & Transmit power & $0.6 \mathrm{~W}$ \\
$P_{R}$ & Receive power & $0.3 \mathrm{~W}$ \\
$P_{I}$ & Idle power & $0.1 \mathrm{~W}$ \\
$P_{S}$ & Sleep power & $0.0 \mathrm{~W}$ \\
$C$ & Channel rate & $2 \mathrm{Mbps}$ \\
$S$ & Source rate & $32 \mathrm{Kbps}$ \\
$N / A$ & Data packet payload & $100 \mathrm{bytes}$ \\
$N / A$ & Data pkt. overhead & 10 bytes \\
$N / A$ & Control Packet size & $10 \mathrm{bytes}$ \\
$N / A$ & Header packet size & 22 bytes \\
$I F S$ & Inter-frame space & $16 \mu \mathrm{s}$ \\
\hline
\end{tabular}

superframe of duration $25.2 \mathrm{~ms}$. Each frame has 7 contention slots and 7 data slots. All the simulations are run for $100 \mathrm{~s}$. We used the energy and propagation models discussed in [12]. In the simulations we used a constant transmit power, which results in a constant transmit radius, $D_{T r}$, of $250 \mathrm{~m}^{1}$, and constant carrier sense range, $D_{C S}$, of $507 \mathrm{~m}$. Acronyms, descriptions and values of the parameters used in the simulations are presented in Table I.

We used the random way-point mobility model for nodes moving within an $800 \mathrm{~m}$ by $800 \mathrm{~m}$ area. Node speeds are chosen from a uniform random distribution between $0.0 \mathrm{~m} / \mathrm{s}$ and $5.0 \mathrm{~m} / \mathrm{s}$ (the average pace of a marathon runner). The pause time is set to zero to avoid non-moving nodes throughout the simulation time. There are 40 mobile nodes in our scenario and the source node is located in the center of the network. This corresponds to a battlefield scenario where the soldiers are on foot (i.e., pedestrian mobility) and the commander of the unit (i.e., a squadron), communicates with the soldiers in the unit in broadcast fashion.

First order quantitative QoS metrics for voice packets are delay and packet delivery ratio [1]. For $32 \mathrm{kbps}$ source coding, the packet delivery ratio should be higher than $90 \%$ in the absence of network delay, and the maximum network delay (excluding the delay contributions by various processing blocks, such as codec assembly and disassembly delays) should be less than $300.0 \mathrm{~ms}$ in the absence of packet loss [13]. In our simulations, we set our QoS objective as $95 \%$ packet delivery ratio and $150 \mathrm{~ms}$

\footnotetext{
${ }^{1} \mathrm{We}$ found that the maximum energy-efficient transmit range for our radio and propagation models is $326.0 \mathrm{~m}$. Thus, our transmission range, which is $250.0 \mathrm{~m}$, is in the energy-efficient range.
}

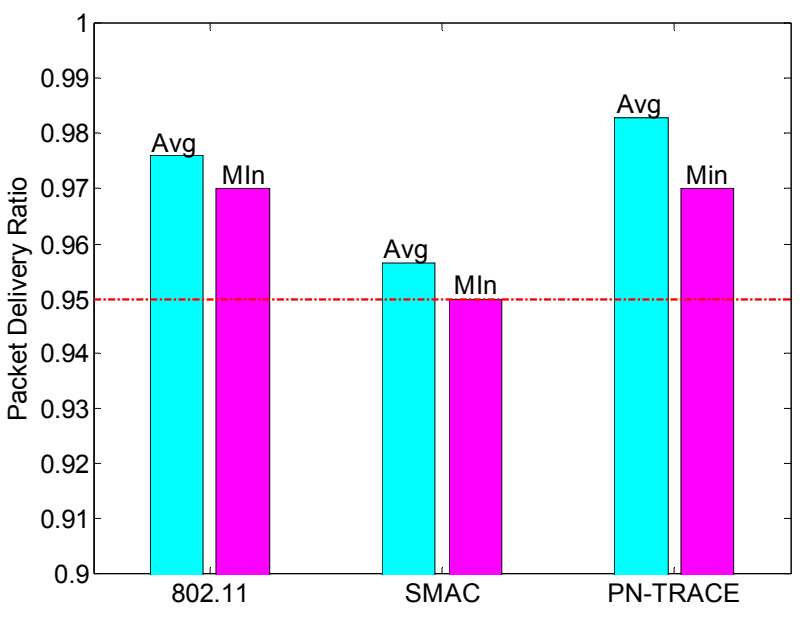

Figure 3. Average and minimum packet delivery ratios for IEEE 802.11, SMAC, and MH-TRACE.

maximum packet delay. Voice packets exceeding $150 \mathrm{~ms}$ delay are dropped at the MAC layer.

\section{Packet Delivery Ratio}

Packet delivery ratio is the ratio of the number of packets generated by the source node to the average number of data packets received by the mobile nodes. The minimum packet delivery ratio is obtained by using the minimum number of packets received. Figure 3 shows the average and minimum packet delivery ratios obtained by using IEEE 802.11-based flooding, SMAC-based flooding, and PN-TRACE. All the packet delivery ratios are very close to each other and above $95 \%$, which is our QoS target for voice packets.

SMAC-based flooding packet delivery ratios are the lowest when compared to IEEE 802.11-based flooding and PN-TRACE. The reason for the lower packet delivery ratio is the sleep/awake periods of SMAC. Actually, the $15 \%$ sleep period corresponds to an effective reduction in bandwidth. For higher sleep percentages, SMAC packet delivery ratio drops below $95 \%$. However, for lower traffic load (i.e., $16 \mathrm{Kbps}$ source rate) SMAC packet delivery ratio is as high as IEEE 802.11 and it is possible to increase the sleep ratio to save more energy.

The difference between IEEE 802.11-based flooding and PN-TRACE is not significant. However, IEEE 802.11 is just on the verge of its unstable operation range; for higher traffic load, its packet delivery ratio will start to drop below $95 \%$. PN-TRACE, on the other hand, is relatively insensitive to changes in the source rate due to the explicit collective channel access mechanism, which prevents PNTRACE from becoming unstable. 
802.11
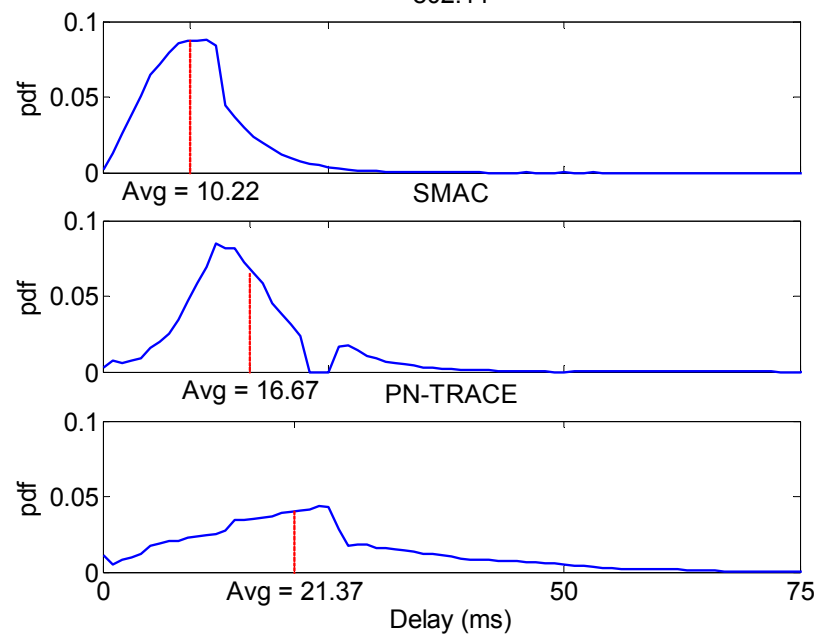

Figure 4. Average packet delay and delay distribution for IEEE 802.11, SMAC and PN-TRACE.

\section{Packet Delay}

We measured the average packet delay and computed the delay distribution for IEEE 802.11-based flooding, SMAC-based flooding, and PN-TRACE, which are presented in Figure 4. Although we set the packet drop threshold to $150 \mathrm{~ms}$, almost all of the received packets have delays less than $75 \mathrm{~ms}$, which shows that the delay is not the bottleneck for QoS in every case (i.e., in our case the bottleneck is the packet delivery ratio). IEEE 802.11based flooding has the lowest packet delay (the average is $10.2 \mathrm{~ms}$ ), and SMAC-based flooding and PN-TRACE follow with $16.7 \mathrm{~ms}$ and $21.4 \mathrm{~ms}$, respectively. SMACbased flooding has higher delay than IEEE 802.11-based flooding due to the sleep times. PN-TRACE delay is higher due to its superframe-based channel access mechanism. Nevertheless, for voice packets all the packets with delay lower than the packet drop threshold are equivalent from the perspective of the application layer.

The PN-TRACE delay distribution is wider than the IEEE 802.11-based flooding delay distribution due to the fact that PN-TRACE packet transmissions and receptions are at regular time instants; whereas IEEE 802.11 packet transmissions and receptions are at arbitrary time instants. The notch in the delay distribution of SMAC-based flooding is due to the sleep periods, when no packets are transmitted.

\section{Energy Dissipation}

The total energy dissipation has four major components: (i) transmit energy dissipation, (ii) receive energy dissipation, (iii) idle energy dissipation, and (iv) carrier sense energy dissipation. The energy dissipation in the carrier sensing mode is the same as the energy dissipation in the receive mode [14].

Figure 5 shows the components of the energy dissipation for IEEE 802.11-based flooding, SMAC-based flooding, and PN-TRACE. IEEE 802.11-based flooding dissipates the highest energy, $213.8 \mathrm{~mJ}$; Since nodes in IEEE 802.11 never enter the sleep mode, even if there were no packet transmissions, nodes would dissipate $100 \mathrm{~mJ}$ (i.e., idle energy dissipation) every second. Interestingly, the major component of the total energy dissipation is carrier sensing, $96.1 \mathrm{~mJ}$ ( $45.0 \%$ of the total energy dissipation). The carrier sensing range, $507 \mathrm{~m}$, is approximately twice the receive range, $250 \mathrm{~m}$. Thus, by assuming the transmissions are approximately evenly distributed in time and space, the average carrier sensing time is three times higher than the average receive time. Furthermore, if the time spent in idle mode (i.e., no transmissions in the carrier sense range) and the time spent in the carrier sense mode were equal, the energy dissipated in carrier sensing would be three times larger than the energy dissipated in the idle mode (i.e., $P_{R} / P_{I}=3$ ).

Receive power, $61.5 \mathrm{~mJ}(28.8 \%)$, is the second largest component of the total energy dissipation for IEEE 802.11-based flooding due to the high redundancy of flooding (i.e., each node retransmits every packet it receives for the first time). Idle energy dissipation, $45.7 \mathrm{~mJ}$ $(21.4 \%)$, is the third largest component of the total energy dissipation. Transmit energy dissipation, $10.5 \mathrm{~mJ}(4.9 \%)$, is the least energy consuming component in the total energy dissipation.

SMAC-based flooding energy dissipation, $206.5 \mathrm{~mJ}$, is

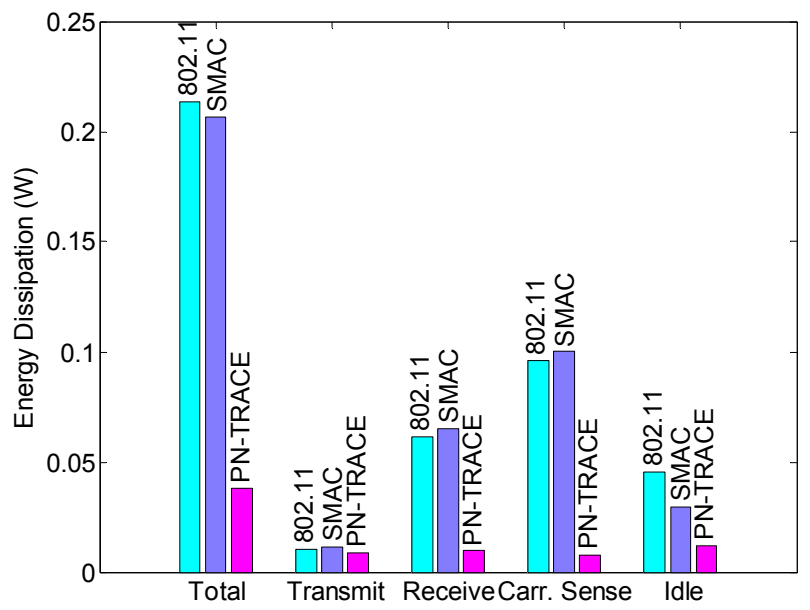

Figure 5. Total, transmit, receive, idle, and carrier sense energy dissipations per node per second for IEEE 802.11-based flooding, SMAC-based flooding, and PN-TRACE. 
$3.5 \%$ less than that of IEEE 802.11-based flooding. The energy savings of SMAC-based flooding is only marginal due to the limited sleep time. For example, for a $50 \%$ sleep ratio, SMAC-based flooding energy dissipation, $137.1 \mathrm{~mJ}$, is $36.0 \%$ less than IEEE 802.11-based flooding. However, in that case the average packet delivery ratio drops to $89 \%$ and the average packet delay increases to $64 \mathrm{~ms}$.

SMAC-based flooding carrier sense, $100.1 \mathrm{~mJ}$ (48.5\%), and receive energy dissipation, $65.4 \mathrm{~mJ}$ (31.7\%), components are slightly larger than IEEE 802.11-based flooding. Idle energy dissipation, $29.6 \mathrm{~mJ}(14.3 \%)$, is slightly lower due to the sleep time energy savings. SMAC-based flooding transmit energy dissipation, $11.4 \mathrm{~mJ}(5.5 \%)$, is similar to that of IEEE 802.11-based flooding.

The total energy dissipation of PN-TRACE, $38.1 \mathrm{~mJ}$, is much lower than the other architectures (i.e., $17.8 \%$ of IEEE 802.11-based flooding energy dissipation and $18.4 \%$ of SMAC-based flooding energy dissipation). However, PN-TRACE energy dissipation is $164.6 \%$ higher than the ideal energy dissipation, $14.4 \mathrm{~mJ}$, which is the energy required for transmitting and receiving each generated bit without any extra energy dissipation, such as carrier sensing and idling mode energy dissipation, and energy dissipated on overhead. All components of the total energy dissipation (transmit, $8.8 \mathrm{~mJ}-23.1 \%$, receive, $9.6 \mathrm{~mJ}-25.2 \%$, carrier sense, $7.7 \mathrm{~mJ}-20.2 \%$, idle, $12.0 \mathrm{~mJ}-31.5 \%$ ) are close to each other and much less than their counterparts in the other schemes except the transmit energy dissipation. Note that in all of the three flooding schemes, all of the nodes are required to retransmit each packet; thus, it is not possible to reduce the transmit energy dissipation in flooding.

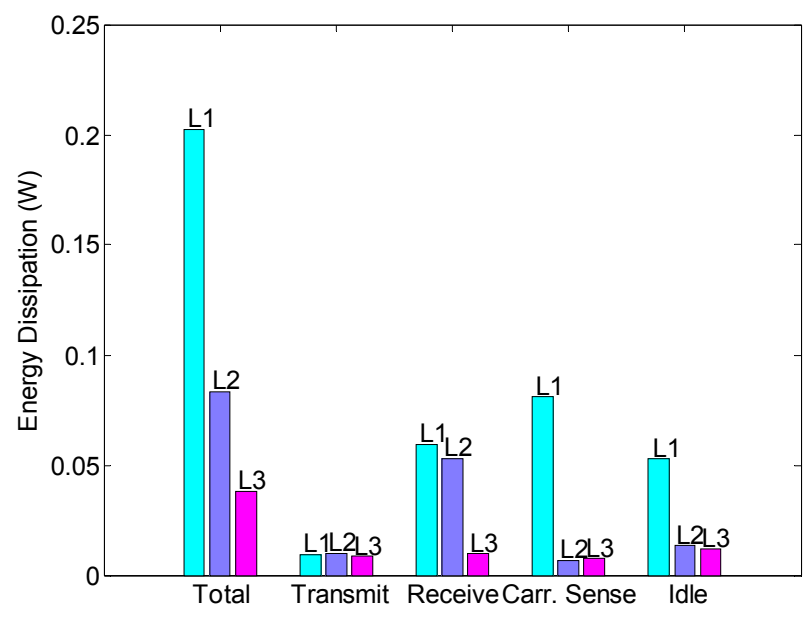

Figure 6. Total, transmit, receive, idle, and carrier sense energy dissipations per node per second for PN-

TRACE L1, PN-TRACE L2, and PN-TRACE L3.

\section{PN-TRACE Energy Dissipation}

To gain better insight into the energy efficiency of PNTRACE, we ran simulations with three versions of PNTRACE. In PN-TRACE L1 (level 1), nodes never enter the sleep mode. In L2 nodes enter the sleep mode whenever they are not involved with control mechanisms, such as beacon transmission/reception or listening for beacon, header, or IS packets, and data transmissions and receptions. However, in L2 all of the data packets are received without discrimination. In L3, any packet that has previously been received is not received again. Each packet has a unique sequence number, which is composed of the source node ID and source node packet sequence number. Each data transmission is preceded by a short IS packet, which contains the data packet's unique ID, thereby allowing the nodes in the receive range to discriminate the packets and only receive one copy of each packet.

Figure 6 shows the components of the energy dissipation for L1, L2, and L3. As expected, L1 energy dissipation, $202.5 \mathrm{~mJ}$, is much higher than L2, $83.5 \mathrm{~mJ}$, and L3, $38.1 \mathrm{~mJ}$, energy dissipations. The difference between L1 and L2 arises from the carrier sensing and idle energy dissipations. In L2 nodes enter the sleep mode instead of wasting energy in idle mode or carrier sensing. The difference between L2 and L3 is due to the receive energy dissipation (i.e., nodes in $\mathrm{L} 2$ receive all the packets in their receive range, whereas nodes in L3 only receive the packets that have not been received before). Transmit energy dissipation is the same for all three PN-TRACE schemes as well as the 802.11 and SMAC schemes. Although L3 energy dissipation is only $18.81 \%$ of L1 and $45.6 \%$ of L2, no information is lost by the energy saving mechanisms (i.e., the packet delivery ratio and packet delay characteristics are identical for L1, L2 and L3).

\section{CONCLUSION}

In this paper we analyzed several approaches for providing network-wide real-time data broadcasting through flooding. We presented the PN-TRACE network architecture, which is designed for energy efficient multihop real-time data broadcasting. We performed extensive simulations to test the performance of PN-TRACE and to compare it with IEEE 802.11 and SMAC-based flooding architectures.

All of the architectures successfully meet the QoS requirements of voice communications (i.e., packet delivery ratio higher than $95 \%$ and maximum packet delay lower than $150 \mathrm{~ms}$ ) under our application scenario. However, we found that the energy dissipation of both IEEE 802.11-based flooding and SMAC-based flooding are more than five times that of PN-TRACE. 
We identified four sources of energy dissipation: (i) transmit energy, (ii) receive energy, (iii) idle energy, and (iv) carrier sense energy. Contrary to common belief that the dominant term in energy dissipation is idle energy dissipation, in all of the architectures we investigated (except PN-TRACE L2 and PN-TRACE L3) the dominant energy dissipation is found to be the carrier sense energy dissipation. The second largest source of energy dissipation is the receive energy. Transmit energy dissipation turns out to be the least important term. Thus, the energy savings by transmit power optimization is marginal (i.e., only $4.9 \%$ of the energy dissipation is due to the transmit energy in IEEE 802.11-based flooding).

PN-TRACE achieves its high energy savings by avoiding energy waste in carrier sensing and in idle mode. Furthermore, data discrimination through information summarization is also shown to be very effective in reducing the receive energy dissipation.

The basic design philosophy of SMAC, which is turning the radios off periodically to avoid idle energy dissipation, is shown to be ineffective for high traffic loads due to the small sleep/awake ratio required to obtain a high packet delivery ratio.

\section{FUTURE WORK}

In this paper we have shown that PN-TRACE is a highly energy-efficient network-wide real-time data broadcasting scheme. However, due to the inherent inefficiency of flooding, PN-TRACE is not scalable to large and/or dense networks, and high traffic loads. Thus, the network layer broadcast routing algorithm should be modified to reinforce the scalability of PN-TRACE.

Network-wide broadcasting algorithms can be classified into three main categories: (i) non-coordinated, (ii) fully coordinated, and (iii) partially coordinated. Flooding is an example of a non-coordinated broadcast algorithm, where nodes rebroadcast without any coordination. The goal of a fully controlled algorithm is to create a Minimum Connected Dominating Set (MCDS), which is the smallest set of rebroadcasting nodes such that the set of nodes are connected and all non-set nodes are within transmit range of at least one member of the MCDS [6]. However, creation of an MCDS is not practical, even with the assumption of global knowledge, due to the NP-hardness of the problem. Partially coordinated broadcast algorithms can be considered as approximate limited scope MCDS's based on one-hop or two-hop neighborhood and/or topology information. The efficiency of these algorithms is better than non-coordinated algorithms and they do not need global information, unlike fully coordinated algorithms.

Instead of using an existing partially coordinated broadcast algorithm, we plan to develop our own partially coordinated broadcast algorithm. Since PN-TRACE already has distributed coordination through clustering, what is needed is a mechanism to select gateways, which forward packets from clusterhead to clusterhead. The selection of gateways will be based on connectivity, where highly connected nodes (i.e., those with a high number of clusterheads in their receive range) will be preferred as gateways to reduce the number of rebroadcasts.

\section{REFERENCES}

[1] D. J. Goodman and S. W. Wei, "Efficiency of packet reservation multiple access," IEEE Trans. Vehic. Technol., vol 40, pp. 170-176, Feb. 1991.

[2] A. Ephremides and T. V. Truong, "Scheduling broadcasts in multihop radio networks," IEEE Trans. Commun., vol. 38, pp. 456-460, 1990.

[3] P.-J. Wan, G. Calinescu, X.-Y. Li, and O. Frieder, "Minimum energy broadcast routing in static ad hoc wireless networks," inProc. IEEE INFOCOM, pp. 11621171, 2001.

[4] P. Chen, B. O'dea, and E. Callaway, "Energy efficient system design with optimum transmission range for wireless ad hoc networks," in Proc. IEEE ICC, Vol. 2, pp. 945-952, 2002.

[5] C. R. Dow, J. H. Lin, A. F. Hwang, and Y. W. Wang, "An efficient distributed clustering scheme for ad-hoc wireless networks," IEICE Trans. Commun., vol. E85-B, pp. 15611571, 2002.

[6] B. Williams and T. Camp, "Comparison of broadcasting techniques for mobile ad hoc networks," in Proc. ACM MOBIHOC, pp 194-205, 2002.

[7] S. Y. Ni, Y. C. Tseng, Y. S. Chen, and J. P. Shen, "The broadcast storm problem in a mobile ad hoc network," in Proc. ACM/IEEE MOBICOM, pp. 151-162, 1999.

[8] D. B. Johnson and D. A. Maltz, "Dynamic source routing in ad hoc wireless networks," Mobile Computing, chapter 5, pp. 153-181, Kluwer Academic Publishers, 1996.

[9] B. Tavli and W. B. Heinzelman, "MH-TRACE: Multi hop time reservation using adaptive control for energy efficiency," in Proc. IEEE MILCOM, pp. 1292-1297, 2003.

[10]B. O'Hara and A. Petrick, The IEEE 802.11 Handbook: A Designer's Companion, IEEE Press, 1999.

[11] W. Ye, J. Heidemann, and D. Estrin, "An energy-efficient MAC protocol for wireless sensor networks," in Proc. IEEE INFOCOM, pp. 1567-1576, 2002.

[12] W. B. Heinzelman, A. Chandrakasan, and H. Balakrishnan, "An application specific protocol architecture for wireless microsensor networks," IEEE Trans. Wireless Comm., vol. 1, pp. 660-670, 2002.

[13] J. Janssen, D. D. Vleeschauwer, G. H. Petit, R. Windey, and J. M. Leroy, "Delay bounds for voice over IP calls transported over satellite access links," Kluwer Mobile Networks and Applications, vol. 7, pp. 79-89, 2002.

[14] D. Qiao, S. Choi, A. Jain, and K. G. Shin, "MiSer: an optimal low-energy transmission strategy for IEEE 802.11a/h," in Proc. MOBICOM, pp. 161-175, 2003. 